\title{
Fatores associados a quedas em idosos: inquérito domiciliar
}

\section{Factors associated with falls in older adults: a household survey \\ Factores asociados con las caídas de mayores: encuesta domiciliaria}

\author{
Samya Regina Nunes Oliveira (iD \\ Universidade Estadual do Ceará - Fortaleza (CE) - Brasil \\ Francisco Marcos de Lima Messias (D) \\ Prefeitura Municipal de Fortaleza - Fortaleza (CE) - Brasil \\ José Auricélio Bernardo Cândido \\ Universidade Estadual do Ceará - Fortaleza (CE) - Brasil
}

Geanne Maria Costa Torres (iD

Universidade Estadual do Ceará - Fortaleza (CE) - Brasil

Inês Dolores Teles Figueiredo (iD

Universidade Regional do Cariri - Crato (CE) - Brasil

Antônio Germane Alves Pinto (iD

Universidade Regional do Cariri - Crato (CE) - Brasil

Maria Rosilene Cândido Moreira (iD

Universidade Federal do Cariri - Crato (CE) - Brasil

Maria Irismar de Almeida iD

Universidade Estadual do Ceará - Fortaleza (CE) - Brasil

\section{RESUMO}

Objetivo: Identificar a prevalência de quedas e fatores associados em idosos no ambiente domiciliar. Métodos: Estudo transversal quantitativo, tipo inquérito domiciliar, observacional e analítico, realizado com 212 idosos atendidos em uma Unidade de Atenção Primária, em Fortaleza, Ceará, que relacionou aspectos demográficos, sociais e clínicos correspondendo ao autorrelato de quedas nos últimos 12 meses. A análise descritiva foi realizada por meio de frequências absolutas e percentuais. A análise inferencial ocorreu no modelo não ajustado para teste da associação entre o desfecho (ocorrência de quedas) e as variáveis associativas por meio do teste do qui-quadrado de Wald, considerando $p<0,20$ como critério de entrada, e, no modelo ajustado, utilizou-se a regressão de Poisson, considerando-se $p<0,05$. Resultados: A prevalência de quedas foi de 63,7\%, com predomínio em pessoas na faixa etária entre 60 e 79 anos de idade $(63,7 \%)$, do sexo feminino $(53,8 \%)$, que usavam tapetes no domicílio $(66,5 \%)$ e apresentavam duas ou mais comorbidades $(41,5 \%)$. Sua ocorrência foi associada ao sexo feminino $(R P=1,96 ; p<0,03)$, com histórico de duas ou mais comorbidades ( $R P=0,407 ; p<0,04)$ e episódios que envolveram tapetes $(R P=1,975 ; p<0,03)$. Conclusão: Encontrou-se elevada prevalência de quedas nos idosos investigados. A identificação dos fatores, a prevenção de comorbidades e a remoção de acessórios escorregadios nos domicílios constituem mudanças que podem ser estimuladas pela abordagem dos profissionais de saúde.

Descritores: Idoso; Fatores de Risco; Prevenção de Acidentes; Atenção Primária à Saúde.

\section{ABSTRACT}

Objective: To identify the prevalence of falls at home among older adults and associated factors. Methods: This quantitative observational and analytical cross-sectional study used a household survey of 212 older adults treated at a Primary Health Care Center in Fortaleza, Ceará, Brazil, to check for relationships of demographic, social and clinical aspects with self-reported falls in the past 12 months. Descriptive analysis was performed using absolute and percentage frequencies. Inferential analysis consisted of an unadjusted model to test the association between the outcome (occurrence of falls) and the associative variables using the Wald Test with a significance threshold set at $p<0.20$ for inclusion in the model. Poisson Regression was used in the adjusted model considering $p<0.05$. Results: The prevalence rate of falls was $63.7 \%$, with a predominance of people aged between 60 and

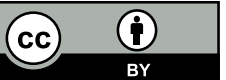


79 years old (63.7\%), women (53.8\%), people who used carpets at home (66.5\%), and people who had two or more comorbidities (41.5\%). The occurrence of falls was associated with female gender ( $P R=1.96 ; p<0.03)$, history of two or more comorbidities $(P R=0.407 ; p<0.04)$, and episodes involving carpets $(P R=1.975 ; p<0.03)$. Conclusion: There was a high prevalence of falls in the older adults analyzed. The identification of factors, the prevention of comorbidities and the removal of slippery accessories from the house are changes that can be encouraged by health professionals.

Descriptors: Aged; Risk Factors; Accident Prevention; Primary Health Care.

\section{RESUMEN}

Objetivo: Identificar la prevalencia de caídas y sus factores asociados en mayores en el ambiente domiciliario. Métodos: Estudio transversal cuantitativo del tipo encuesta domiciliaria, observacional y analítico con 212 mayores asistidos en una Unidad de Atención Primaria de Fortaleza, Ceará, el cual ha relacionado los aspectos demográficos, sociales y clínicos de los relatos de caídas en los últimos 12 meses. El análisis descriptivo ha sido realizado a través de las frecuencias absolutas y porcentuales. El análisis inferencial se dio con el modelo no ajustado para la prueba de asociación entre el resultado (ocurrencia de caídas) y las variables asociativas a través de la prueba de chi-cuadrado de Wald, considerando $p<0,20$ como el criterio de entrada y, para el modelo ajustado, se utilizó la regresión de Poisson considerándose $p<0,05$. Resultados: La prevalencia de las caídas ha sido del 63,7\% con el predominio de personas en la franja de edad entre 60 y 79 años de edad (63,7\%), del sexo femenino (53,8\%), que usaban alfombras en el domicilio $(66,5 \%)$ y que presentaban dos o más comorbilidades $(41,5 \%)$. Su ocurrencia se ha asociado con el sexo femenino $(R P=1,96 ; p<0,03)$, el histórico de dos o más comorbilidades $(R P=0,407 ; p<0,04)$ y los episodios con alfombras $(R P=1,975 ; p<0,03)$. Conclusión: Se ha encontrado una elevada prevalencia de caídas en los mayores investigados. La identificación de los factores, la prevención de las comorbilidades y la remoción de los accesorios resbaladizos en los domicilios son cambios que se puede estimular a través del abordaje de los profesionales sanitarios.

Descriptores: Anciano; Factores de Riesgo; Prevención de Accidentes; Atención Primaria de Salud.

\section{INTRODUÇÃO}

As quedas em idosos são reconhecidas como importante problema de saúde pública, estando relacionadas a fatores de risco que podem levar a incapacidades, lesões e morte. São consideradas como síndromes geriátricas, que afetam as condições das pessoas de gerir suas próprias vidas em consequência de incapacidades físicas, psicológicas, sociais e econômicas ${ }^{(1)}$. Por definição, as quedas estão associadas, de uma forma geral, ao deslocamento, de forma não intencional, do corpo, quando não se encontra o apoio que se busca, e são ocasionadas por circunstâncias multifatoriais, podendo resultar em danos físicos e psicológicos ${ }^{(2)}$.

De acordo com a Organização Mundial de Saúde (OMS), pessoas acima de 65 anos de idade e que sofreram algum episódio de queda por ano têm uma prevalência de $28 \%$ a $35 \%$; em idosos acima de 70 anos de idade, esta varia de $32 \%$ a $42 \%{ }^{(3)}$. Em dados oficiais do Ministério da Saúde do Brasil, por meio de atendimento no Sistema Único de Saúde (SUS), no ano de 2013, foram registrados 93.312 internamentos de pessoas acima de 60 anos que sofreram queda, sendo que, dos registros mencionados, 8.775 chegaram a óbito por essa causa ${ }^{(4)}$. A queda é, no Brasil, um dos principais motivos de internação e causa de morte acidental de pessoas acima de 65 anos, além de ser uma das importantes causas de morbimortalidade, superando as doenças prevalentes para a idade, como as doenças crônicas não transmissíveis ${ }^{(5)}$.

A morbimortalidade relacionada a quedas de idosos é um tema de grande relevância, porém os registros desses agravos ainda não estão sendo realizados de forma satisfatória ou são subnotificados ${ }^{(6)}$.

Em estudos internacionais, encontrou-se prevalência de registro de queda relacionado a idosos em $21 \%$ na Ásia Oriental( ${ }^{(7)}$ e 19,1\% na Polônia ${ }^{(8)}$. No Brasil, podem-se evidenciar estudos apresentando diferentes prevalências de registro para o mesmo agravo em diferentes municípios, como Natal $(68,2 \%)$, Uberaba $(28,3 \%)^{(9)}$, Florianópolis $(18,9 \%)^{(10)}$, São Carlos $(27,6 \%)^{(11)}$, Cuiabá $(37,5 \%)^{(12)}$, Sete Lagoas $(30 \%)^{(13)}$ e Catanduva $(51 \%)^{(14)}$.

As capitais brasileiras apresentaram os maiores registros de quedas de pessoas com 60 anos de idade ou mais, seja por registro de óbito, seja por internamento associado a esse agravo. Os maiores registros relacionados à mortalidade foram em Vitória, Goiânia, Florianópolis e Porto Velho, que apresentaram as maiores taxas de mortalidade por quedas. Já as capitais com maiores taxas de internação foram São Paulo, Natal, Belo Horizonte e Porto Alegre. Entre as regiões brasileiras, a Sudeste, a Nordeste e a Centro-Oeste, seguidas pela Sul e Norte, foram as mais representativas para esse agravo(15).

No Ceará, estudo analisando artigos científicos e entrevistando 50 idosos constatou que $53,8 \%$ dos trabalhos relacionaram as inadequações do ambiente como sendo o principal fator de risco para quedas. Nas entrevistas, 
observou-se que $42 \%$ dos entrevistados havia tido um episódio de queda nos dois últimos anos e que $57 \%$ destas estavam relacionadas ao ambiente doméstico ${ }^{(16)}$.

Percebe-se, no entanto, que 60 a $70 \%$ dos registros de quedas de idosos ocorrem no próprio domicílio ou em seus arredores, e que quanto maior a idade maior o risco, principalmente para pessoas acima de 75 anos ${ }^{(17)}$. Portanto, entende-se que é necessário o reconhecimento dos fatores de risco para se desenvolver medidas de prevenção e promoção da saúde na comunidade e nos domicílios.

Existem os fatores de risco intrínsecos, que estão relacionados diretamente com a pessoa idosa, como alterações fisiológicas do ciclo vital, patologias, consumo de medicamentos e redução da capacidade funcional; e os fatores de risco extrínsecos, que se relacionam às condições do meio ambiente, como a exposição a pisos deslizantes e tapetes, ambientes mal iluminados, ausência de corrimão, mobília instável e presença de animais ${ }^{(18)}$. Já entre idosos institucionalizados, as quedas estavam relacionadas a doenças e ao uso de medicamentos, sendo o principal local de queda o ambiente em que viviam, como o quarto, o corredor e o banheiro ${ }^{(19)}$.

Cada vez mais ferramentas estão sendo utilizadas precocemente para estratificar o risco de adoecer das pessoas, nas diversas patologias, em seus diferentes ciclos de vida, com o intuito de identificá-los precocemente, ainda em nível primário, facilitando o planejamento e o desenvolvimento de ações de prevenção e promoção da saúde da população(20).

Nesse sentido, entende-se que as ações de prevenção e promoção da saúde no contexto da Atenção Primária à Saúde, relacionada aos fatores de risco que possam levar à queda em idosos, ainda são incipientes, e que essas atividades devem estar contidas no planejamento estratégico dos processos de trabalho das equipes da Estratégia Saúde da Família. É por meio da promoção da saúde, em busca de se construir espaços saudáveis, que se permite adotar medidas de educação em saúde sob a ótica e o entendimento dos problemas de saúde, para promover as soluções possíveis ${ }^{(21)}$.

Este estudo encontra sustentação no incentivo da OMS à ampliação do conhecimento dos preditores de quedas em idosos, por entender ser este um dos pilares do modelo de prevenção de quedas. Nesse sentido, o objetivo desta pesquisa foi identificar a prevalência de quedas e os fatores de risco que estão associados a quedas em idosos no ambiente domiciliar.

\section{MÉTODOS}

Estudo de abordagem quantitativa, transversal, observacional e analítico do tipo inquérito domiciliar. Buscou-se seguir de forma descritiva, clara, concisa e coerente todas as etapas da investigação, descrição e análise do objeto, conforme os padrões dos estudos observacionais e as recomendações do guidelines Strengthening The Reporting of Observational studies in Epidemiology (STROBE) ${ }^{(22)}$.

O campo de pesquisa foi a Unidade de Atenção Primária em Saúde (UAPS) localizada na área de abrangência da Secretaria Regional IV, que possui uma população com cerca de 51.160 habitantes. A UAPS atende às comunidades dos bairros Dendê, Itaperi e parte da Serrinha, estando situada no bairro Itaperi. A equipe de Saúde da Família selecionada é denominada Serrinha 5 e possui uma população idosa cadastrada de 330 pessoas, sendo 183 do sexo feminino e 147 do sexo masculino.

Elencaram-se como critérios de inclusão: ter 60 anos ou mais de idade, ser residente na área da abrangência e ser atendido na UAPS. Como critérios de exclusão: qualquer condição de saúde que pudesse gerar viés no estudo, como apresentar déficit cognitivo grave, demência, doença de Parkinson em estágio grave ou em estágio terminal. Após a aplicação dos critérios, a amostra foi totalizada em 212 idosos, sendo 135 mulheres e 77 homens.

A coleta dos dados ocorreu nos domicílios dos idosos, no período de fevereiro a maio de 2018, por meio de entrevista semiestruturada, isto é, contendo questões fechadas e abertas, relacionadas aos aspectos demográficos, sociais e clínicos que envolvem os fatores intrínsecos e extrínsecos, correspondendo ao autorrelato de quedas nos últimos 12 meses. Para realização das entrevistas, contou-se com o enfermeiro da ESF, uma agente comunitária de saúde (ACS), dois estudantes do curso de Medicina e uma professora da mesma universidade, que orientou, por meio de um treinamento, a abordagem aos idosos e a respeito das questões éticas envolvidas na pesquisa.

Este estudo apresentou como variável desfecho: ocorrência de quedas (sim ou não). As variáveis explicativas foram distribuídas em: Sociodemográfica: sexo, idade, estado civil, anos de estudo e renda; Ambiental: características da moradia (estado da rua, presença de jardins, tipo de construção, tipo de moradia) e do ambiente domiciliar (número de cômodos, presença ou não de escadas, tipo de pisos, sistema de prevenção, características da iluminação e banheiros, presença ou não de animais, tipo de animais e local de habitação); Estado de Saúde: uso ou não de medicamentos (quantidade de comprimidos, tipo de medicamentos), uso ou não de tabaco, uso ou não de álcool, 
presença ou não de comorbidades, prática ou não de atividade física; Acesso ao serviço de saúde: transporte utilizado, quantas vezes procurou atendimento, realizou ou não cirurgias prévias, acesso ou não a orientações sobre quedas; e, por fim, Informações sobre quedas: ocorrência ou não de quedas, quantas vezes sofreu queda, qual o motivo da queda, local da queda, presença ou não de fratura, presença ou não de outro tipo de trauma após queda; tipo de trauma pós-queda; procura ou não de atendimento médico, realizou ou não internamento, tomou ou não medidas de prevenção de acidentes após a queda; realizou ou não fisioterapia.

Realizou-se um diário de campo a partir da observação do ambiente residencial, cuja finalidade era realizar anotações referentes às impressões subjetivas do pesquisador (percepções, reações, sentimentos etc.). Após a coleta, os dados foram tabulados no programa Excel, versão 11.0, sendo analisados no software Statistical Package for Social Science for Windows (SPSS), versão 23.0.

Realizou-se a análise descritiva por meio das frequências absolutas e percentuais. Na análise inferencial não ajustada, testou-se a existência de associação entre o desfecho (ocorrência de quedas, não ocorrência de quedas) e as variáveis associativas, por meio do teste do qui-quadrado de Wald, considerando-se como critério de entrada o nível descritivo $p<0,20$. No modelo ajustado de regressão múltipla, utilizou-se a regressão de Poisson, considerandose apenas as variáveis significativas $(p<0,05)$.

O estudo seguiu as determinações contidas na Resolução n..$^{\circ} 466$ de 12 de dezembro de 2012, o qual versa sobre Diretrizes e Normas Regulamentadoras de Pesquisa envolvendo Seres Humanos. O estudo foi aprovado pelo Comitê de Ética sob o Parecer n. ${ }^{\circ} 2.482 .985$.

\section{RESULTADOS}

Entrevistaram-se 212 idosos, sendo 135 do sexo feminino e 77 do masculino. Em relação às variáveis sociodemográficas, a maioria dos entrevistados tinha entre 60 e 79 anos $(n=179 ; 84,5 \%)$, sexo feminino $(n=135$; $63,7 \%)$, sem cônjuge ( $n=114 ; 53,8 \%$ ), renda familiar ( $n=202 ; 95,3 \%)$ e estava aposentada $(n=125 ; 58,9 \%)$.

Em relação às características da moradia, $83,9 \%$ dos participantes morava em ruas em estado de conservação precário, em casas com mais de cinco cômodos, pisos inadequados e com tapetes. Em 98,5\% dos domicílios havia iluminação adequada e os ambientes da casa eram de fácil acesso, porém não havia barras de apoio ou cadeiras adaptadas para banho. Pouco mais da metade $(54,9 \%)$ dos participantes possuía animais em casa.

Sobre o estado de saúde, $86,3 \%$ dos idosos informaram a utilização de medicamentos para tratamento de saúde, histórico de comorbidades e cirurgias anteriores; $68,3 \%$ recorreram à unidade básica para suas necessidades de saúde e 97,2\% afirmaram nunca terem recebido orientações sobre quedas (Figura 1).

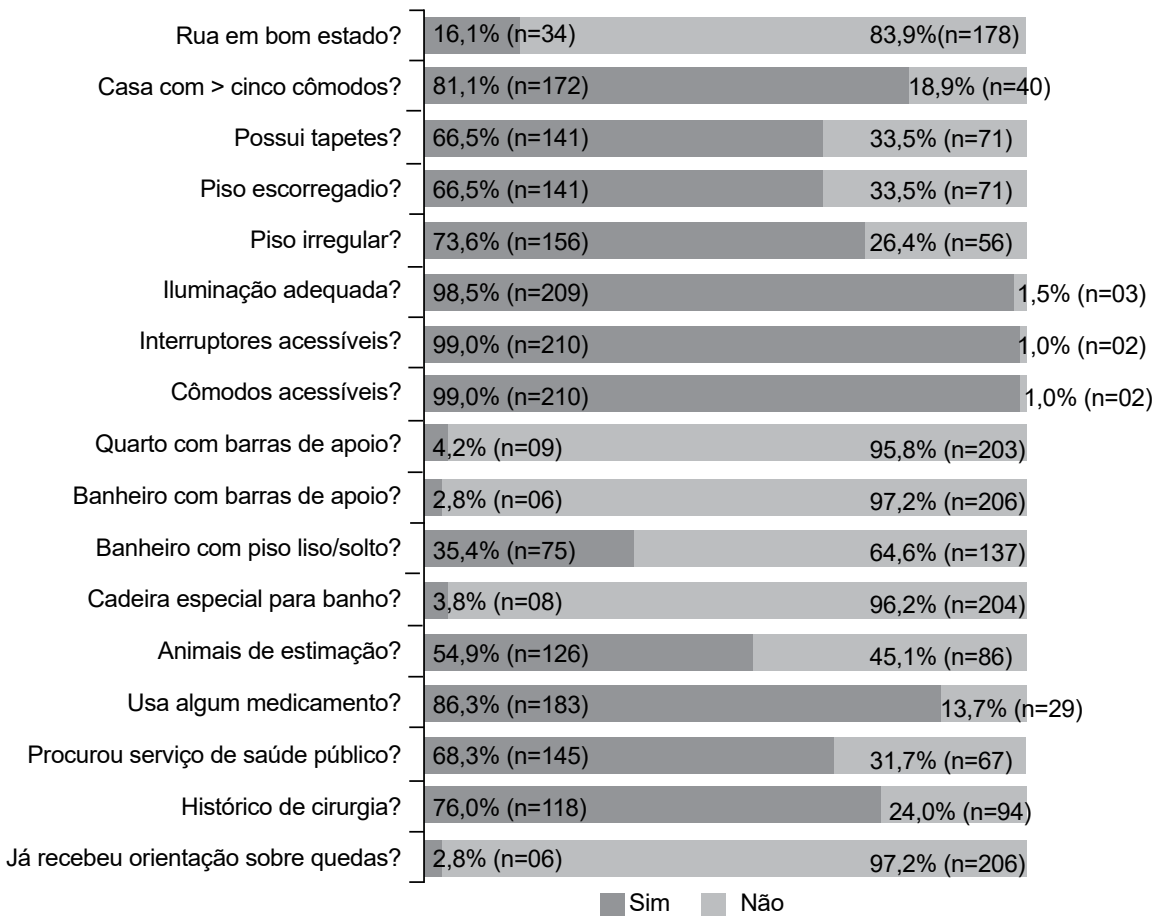

Fonte: própria pesquisa (2018)

Figura 1 - Características da moradia, estado de saúde e acesso ao serviço de saúde da comunidade pelos idosos participantes do estudo. Fortaleza, 2018. 
Após análise estatística no modelo não ajustado $(p<0,20)$, relacionando-se a ocorrência ou não de quedas em idosos com as variáveis associativas, identificaram-se as variáveis: sexo, estado conjugal, uso de tapetes, tipo de piso, uso de barras de apoio e estado de saúde (comorbidades), estatisticamente significantes (Tabela I).

Tabela I - Ocorrência de quedas em relação às variáveis associativas no modelo não ajustado. Fortaleza, Ceará, 2020.

\begin{tabular}{|c|c|c|c|c|c|c|c|c|c|}
\hline \multirow[t]{2}{*}{ Variáveis } & & \multicolumn{5}{|c|}{ Quedas } & \multirow[b]{2}{*}{ RP } & \multirow[b]{2}{*}{ IC 95\% } & \multirow[b]{2}{*}{$p$} \\
\hline & & $\mathbf{n}$ & Sim & $\%$ & Não & $\%$ & & & \\
\hline \multirow[t]{2}{*}{ Sexo } & Feminino & 135 & 96 & 71,1 & 39 & 28,9 & 2,398 & $1,341-4,290$ & 0,003 \\
\hline & Masculino & 77 & 39 & 50,6 & 38 & 49,4 & & & \\
\hline \multirow[t]{2}{*}{ Estado conjugal } & Com companheiro & 98 & 57 & 58,2 & 41 & 41,8 & 1,13 & $0,951-1,343$ & 0,163 \\
\hline & Sem companheiro & 114 & 78 & 68,4 & 36 & 31,6 & & & \\
\hline \multirow[t]{2}{*}{ Uso de tapetes } & Sim & 141 & 97 & 68,8 & 44 & 31,2 & 0,522 & $0,290-0,939$ & 0,003 \\
\hline & Não & 71 & 38 & 53,5 & 33 & 46,5 & & & \\
\hline \multirow[t]{3}{*}{ Tipo de piso } & Cerâmica & 161 & 106 & 65,8 & 55 & 34,2 & 3,81 & $0,917-3,673$ & 0,116 \\
\hline & Cimento & 41 & 21 & 51,2 & 20 & 48,8 & & & \\
\hline & Outros & 10 & 8 & 80 & 2 & 20 & & & \\
\hline \multirow[t]{2}{*}{ Uso de barras de apoio } & Sim & 09 & 08 & 88,9 & 01 & 11,1 & 5,015 & $0,556-45,554$ & 0,151 \\
\hline & Não & 203 & 127 & 62,6 & 76 & 37,4 & & & \\
\hline \multirow[t]{2}{*}{ Estado de saúde } & Uma comorbidade & 124 & 69 & 55,6 & 55 & 44,4 & & & \\
\hline & Mais de uma comorbidade & 88 & 66 & 75 & 22 & 25 & 0,418 & $0,230-0,761$ & 0,004 \\
\hline
\end{tabular}

RP: razão de prevalência; IC 95\%: intervalo de 95\% de confiança. p: teste de associação qui-quadrado

No modelo multivariado ajustado a partir da regressão de Poisson, ao nível de significância $p<0,05$, houve significância estatística na análise das variáveis: sexo, uso de tapetes e comorbidades. Obteve-se razão de prevalência do sexo feminino 1,960 vezes em relação ao sexo masculino, razão de prevalência do uso de tapetes 1,975 vezes em relação ao não uso de tapetes e a razão de prevalência de mais de uma comorbidade 0,407 vezes em relação a uma comorbidade. Portanto, encontrou-se, neste estudo, o fator de proteção para quedas em idosos: ser do sexo masculino, não utilizar tapetes e ter apenas uma comorbidade (Tabela II).

Tabela II - Ocorrência de quedas em relação às variáveis associativas no modelo multivariado ajustado. Fortaleza, Ceará, 2020.

\begin{tabular}{lcccc}
\hline Variáveis & & RP & IC 95\% & p \\
\hline Sexo & $\begin{array}{l}\text { Feminino } \\
\text { Masculino }\end{array}$ & 1,960 & $1,014-3,789$ & 0,045 \\
Uso de tapetes & $\begin{array}{c}\text { Usa tapetes } \\
\text { Não usa tapetes } \\
\text { Estado de saúde }\end{array}$ & 1,975 & $1,38-3,760$ & 0,038 \\
& $\begin{array}{c}\text { Mais de uma comorbidade } \\
\text { Uma comorbidade }\end{array}$ & 0,407 & $0,217-0,764$ & 0,004 \\
\hline
\end{tabular}

RP: razão de prevalência; IC 95\%: intervalo de 95\% de confiança. p: teste de associação qui-quadrado

\section{DISCUSSÃO}

No presente estudo, após análise estatística final, a partir do modelo multivariado ajustado, houve significância estatística na análise dos fatores de risco: sexo, uso de tapetes e comorbidades. Percebeu-se uma alta prevalência em relação ao sexo feminino e chance de quedas quase duas vezes maior em relação ao sexo masculino.

A identificação do sexo feminino como fator de risco para ocorrência de quedas pode estar relacionada à maior exposição a fatores domésticos, a alterações clínicas, a condições de adoecimento decorrente de uma menor força muscular e, ainda, ao processo de adoecimento crônico. Alguns estudos apontam uma associação de quedas em mulheres com idade mais avançada, com morbidades, com vivência sem companheiro/a, com menor escolaridade e com uso de medicamentos ${ }^{(23,24)}$. 
Outro fator de risco evidenciado foi o uso de tapetes, de forma que se percebeu que o uso desse objeto causa duas vezes mais quedas em idosos do que o não uso. Outro estudo evidenciou que os fatores extrínsecos, como o tapetes, estão associados às condições inadequadas do ambiente no trânsito da pessoa idosa, ocasionando quedas dentro de casa ou em suas adjacências durante o desempenho de suas atividades diárias, como caminhar ou ir ao banheiro, principalmente no período noturno ${ }^{(25)}$.

Corroborando esses estudos, recomenda-se que, como medida de prevenção, os tapetes e carpetes possam ser evitados, principalmente no ambiente doméstico. Caso haja necessidade da utilização, devem estar bem fixados e esticados, de preferência colados ${ }^{(26)}$. Percebe-se que as medidas preventivas ainda são pouco implementadas no ambiente domiciliar, decorrente da falta de informações em relação à família e à falta de educação em saúde preventiva por parte dos profissionais de saúde ${ }^{(27)}$.

O estado de saúde dos idosos foi outro fator de risco intrínseco que se apresentou de modo significativo, sendo que, neste estudo, as pessoas que apresentaram mais de uma comorbidade tiveram mais chance de quedas do que as pessoas que apresentaram apenas uma comorbidade.

As comorbidades, como as doenças cardiovasculares, hipertensão arterial, diabetes mellitus, doenças pulmonares, câncer, derrame cerebral e osteoporose, presentes em grande parte das pessoas idosas, predispõem a um maior risco de quedas, levando a uma maior vulnerabilidade no ambiente domiciliar, mas também no ambiente hospitalar, o que pode comprometer a capacidade funcional, a autonomia e a qualidade de vida dos idosos ${ }^{(28)}$.

Ainda na análise final, alguns fatores de risco não apresentaram significância estatística como o estado conjugal, o tipo de piso e uso de barras de apoio. Porém, tais fatores merecem atenção voltada para a prevenção e ações de promoção da saúde no domicílio.

Em relação ao estado conjugal, o fato de morar sozinho pode acarretar maior risco de quedas devido ao idoso não poder dividir as tarefas do cotidiano ou não ter ajuda para desenvolver atividades pessoais ${ }^{(29)}$.

Os fatores de risco relacionados às condições ambientais, ao tipo de piso e ao uso de barras de apoio também interferem no risco de quedas, sendo necessário um investimento financeiro para a implementação de medidas preventivas que implicam nas condições sociais das pessoas idosas e de seus familiares ${ }^{(30)}$.

Algumas limitações puderam ser observadas durante este estudo, tais como: a dificuldade de acesso ao interior do domicílio, decorrente da desconfiança da família em participar da pesquisa; ter sido realizado em uma comunidade assistida por apenas uma equipe de Saúde da Família, o que pode refletir uma realidade peculiar, embora outros estudos, em outras regiões, apontem resultados semelhantes ao que foi encontrado aqui.

Entretanto, entendendo que as quedas em idosos são de origem multifatorial, ressalta-se que as medidas de prevenção e promoção de saúde nessa faixa etária requerem ações multiprofissionais e multidisciplinares, destacando-se que, dos fatores de risco identificados neste estudo, a prevenção de comorbidades e a remoção de acessórios escorregadios nos domicílios constituem mudanças que podem ser estimuladas pela abordagem dos profissionais de saúde.

\section{CONCLUSÃO}

Encontrou-se a prevalência de quedas de $63,7 \%$ nos idosos investigados, cuja ocorrência deu-se no ambiente domiciliar, no qual o sexo feminino, a existência de duas ou mais comorbidades e a presença de tapetes no interior da residência consistiram em fatores de risco a elas associados.

\section{CONFLITOS DE INTERESSE}

O manuscrito não apresenta relações que possam implicar em potenciais conflitos de interesses.

\section{CONTRIBUIÇÕES}

Todos os autores participaram da concepção e delineamento do estudo, da análise e interpretação dos dados, da redação ou revisão crítica relevante do conteúdo intelectual do manuscrito, da aprovação final da versão a ser publicada e aprovaram a versão final encaminhada, de modo que todos têm responsabilidade por todos os aspectos do trabalho, incluindo a garantia de sua precisão e integridade.

\section{REFERÊNCIAS}


1. Wingerter DG, Azevedo UN, Marcaccini AM, Alves MSCF, Ferreira MAF, Moura LKB. Produção científica sobre quedas e óbitos em idosos: Uma análise bibliométrica. Rev Bras Geriatr Gerontol [Internet]. 2018 [acesso em 2020 Mar 2];21(3):320-9. Disponível em: https://www.scielo.br/scielo.php?script=sci_ abstract\&pid=S1809-98232018000300320\&lng=en\&nrm=iso\&tlng=pt

2. Sociedade Hospital Samaritano. Manual de Segurança do Paciente [Internet]. São Paulo: Sociedade Hospital Samaritano; 2016 [acesso em 2020 Mar 2]. Disponível em: http://www.samaritano.med.br/paginas/manualde-internacao/

3. Tako KV, Andrade LC, Marinho HML, Neves VS, Santos AE, Lopes MS, et al. Profile and prevalece of falls in elderly perfil y prevalência de caidas en idosos. Rev Enferm UFPE [Internet]. 2017 [acesso em 2020 Mar 2];11(Supl.11):4687-91. Disponível em: https://periodicos.ufpe.br/revistas/revistaenfermagem/article/ download/231210/25216

4. Ministério da Saúde (BR). Datasus: informações de Saúde [Internet]. Brasília: Ministério da Saúde; 2015 [acesso em 2020 Mar 2]. Disponível em: http://www2.datasus.gov.br/DATASUS/index.php?area=0203

5. Freitas MG, Bonolo PF, Moraes EM, Machado CJM. Idosos atendidos em serviços de urgência no Brasil: um estudo para vítimas de quedas e de acidentes de trânsito. Ciênc Saúde Colet [Internet]. 2015 [acesso em 2020 Mar 2];20(3):701-12. Disponível em: https://www.scielo.br/scielo.php?pid=S1413$81232015000300701 \&$ script=sci_abstract\&tIng=pt

6. Messias KLM, Bispo JP Jr, Pegado MFQ, Oliveira LC, Peixoto TG, Sales MAC, et al. Qualidade da informação dos óbitos por causas externas em Fortaleza, Ceará, Brasil. Ciênc Saúde Colet [Internet]. 2016 [acesso em 2020 Mar 2];21(4):1255-67. Disponível em: https://www.scielo.br/scielo.php?script=sci_ arttext\&pid=S1413-81232016000401255\&lng=pt\&nrm=iso

7. Wu TY, Chie WC, Yang RS, Liu JP, Kuo KL, Wong WK, et al. Factors associated with falls among communitydwelling older people in Taiwan. Ann Acad Med Singapore [Internet]. 2013 [acesso em 2020 Mar 2];42(7):320-7. Disponível em: https://pubmed.ncbi.nlm.nih.gov/23949261/

8. Skalska A, Wizner B, Piotrowicz K, Klich-Raczka A, Klimek E, Mossakowska H, et al. The prevalence of falls and their relation to visual and hearing impairments among a nation-wide cohort of older Poles. Rev Exp Gerontol [Internet]. 2013 [acesso em 2020 Mar 2];48(2):140-6. Disponível em: https://www.scielo.br/scielo. php?pid=S0104-07072016000200312\&script=sci_arttext\&tlng=en

9. Nascimento JS, Tavares DMS. Prevalência e fatores associados a quedas em idosos. Texto \& Contexto Enferm [Internet]. 2016 [acesso em 2020 Mar 2];25(2):e0360015. Disponível em: https://www.scielo.br/pdf/ tce/v25n2/0104-0707-tce-25-02-0360015.pdf

10. Antes D, Schneider JC, Benedetti TRB, D'ors E. Medo de queda recorrente e fatores associados em idosos de Florianópolis, Santa Catarina, Brasil. Cad Saude Pública [Internet]. 2013 [acesso em 2020 Mar 2];29(4):758-68. Disponível em: https://www.scielo.br/scielo.php?script=sci_arttext\&pid=S0102311X2013000400013\&lang=pt

11. Aveiro MC, Driusso P, Barham EJ, Pavarini SCL, Oishi J. Mobilidade e risco de quedas de população idosa da comunidade de São Carlos. Ciênc Saúde Colet [Internet]. 2012 [acesso em 2020 Mar 2];17(9):2481-8. Disponivel em: https://www.scielo.br/scielo.php?pid=\$1413-81232012000900028\&script=sci_abstract\&tlng=pt

12. Soares WJS, Moraes SA, Ferriolli E, Perracini MR. Fatores associados a quedas e quedas recorrentes em idosos: estudo de base populacional. Rev Bras Geriatr Gerontol [Internet]. 2014 [acesso em 2020 Mar 2];17(1):49-60. Disponível em: https://www.scielo.br/pdf/rbgg/v17n1/1809-9823-rbgg-17-01-00049.pdf

13. Campos ACV, Ferreira EF, Vargas AM. Determinantes do envelhecimento ativo segundo a qualidade de vida e gênero. Ciênc Saúde Colet [Internet]. 2015 [acesso em 2020 Mar 2];20(7):2221-37. Disponível em: https:// www.scielo.br/scielo.php?pid=S1413-81232015000702221\&script=sci_abstract\&tlng=pt

14. Silva LT, Martinez EZ, Manco ARX, Souza AP Jr, Arruda MF. A Associação entre a ocorrência de quedas e a alteração de equilíbrio e marcha em idosos. Rev Saúde Pesqui [Internet]. 2014 [acesso em 2020 Mar 2];7(1):25-34. Disponível em: https://www.scielo.br/pdf/tce/v25n2/0104-0707-tce-25-02-0360015.pdf

15. Abreu DROM, Novaes ES, Oliveira RR, Mathias TAF, Marcon SS. Internação e mortalidade por 
quedas em idosos no Brasil: análise de tendência. Ciênc Saúde Colet [Internet]. 2018 [acesso em 2020 Mar 2];23(4):1131-41. Disponível em: https://www.scielo.br/scielo.php?script=sci_arttext\&pid $=$ S1413-8123201800040113

16. Cavalcante ALP, Aguiar JB, Gurgel LA. Fatores associados a quedas em idosos residentes em um bairro de Fortaleza, Ceará. Rev Bras Geriatr Gerontol [Internet]. 2012 [acesso em 2020 Mar 2];15(1):137-46. Disponível em: https://www.scielo.br/scielo.php?script=sci_arttext\&pid=S1809-98232012000100015

17. Souza AQ, Pegorari MS, Nascimento JS, Oliveira PB, Tavares DMS. Incidência e fatores preditivos de quedas em idosos na comunidade: um estudo longitudinal. Ciênc Saúde Colet [Internet]. 2019 [acesso em 2020 Mar 2];24(9):3507-16. Disponível em: https://www.scielo.br/scielo.php?script=sci_arttext\&pid $=\mathrm{S} 1413-81232019000903507$

18. Gautério DP, Zortea B, Santos SSC, Tarouco BS, Lopes MJ, Fonseca CJ. Riscos de novos acidentes por quedas em idosos atendidos em ambulatório de traumatologia. Invest Educ Enferm [Internet]. 2015 [acesso em 2020 Mar 2];33(1):35-43. Disponível em: http://www.scielo.org.co/scielo.php?pid=S0120$53072015000100005 \&$ script $=$ sci_arttext\&tlng=pt

19. Ferreira LMB, Macedo R, Karyna MOBF, Jerez-Roig J, Araújo JRT, Lima KC. Quedas recorrentes e fatores de risco em idosos institucionalizados. Ciênc Saúde Colet [Internet]. 2019 [acesso em 2020 Mar 2];24(1):67-75. Disponível em: https://www.scielo.br/scielo.php?pid=S1413-81232019000100067\&script=sci_abstract\&tlng=pt

20. Cândido JAB, Torres GMC, Figueiredo IDT, Morais APP, Pinto FJM, Pinto AGA, et al. FINDRISK: estratificação do risco para diabetes mellitus na saúde coletiva. Rev Bras Promoç Saúde [Internet]. 2017 [acesso em 2020 Mar 2];30(3):1-8. Disponível em: https://periodicos.unifor.br/RBPS/article/view/6118

21. Monken $M$, Barcellos $C$. O território na promoção e vigilância em saúde [Internet]. Rio de Janeiro: Fiocruz; 2016 [acesso em 2020 Mar 2]. Disponível em: https://www.arca.fiocruz.br/bitstream/icict/39206/2/O\%20 territ\%C3\%B3rio\%20e\%20o\%20processo\%20sa\%C3\%BAde-doen\%C3\%A7a\%20-\%200\%20 Territ\%C3\%B3rio\%20na\%20Promo\%C3\%A7\%C3\%A3o\%20e\%20Vigil\%C3\%A2ncia\%20em\%20 Sa\%C3\%BAde.pdf

22. Malta M, Cardoso LO, Bastos FI, Magnanini MMF, Silva CMFP. Iniciativa STROBE: subsídios para a comunicação de estudos observacionais. Rev Saúde Pública [Internet]. 2010 [acesso em 2020 Mar 2];44(3):559-65. Disponível em: http://www.scielo.br/scielo.php?script=sci_arttext\&pid=S0034$89102010000300021 \&$ lng $=$ en\&nrm=iso

23. Nascimento MM. Factores predictores de caídas en mujeres que practican actividad física. Rev Bras Promoç Saúde [Internet]. 2017 [acesso em 2020 Mar 2];30(3):1-7. Disponível em: https://periodicos.unifor.br/RBPS/ article/view/6302/pdf

24. Meirelles RC Jr, Castro JO, Faria LR, Silva CLA, Alves WA. Notificações de óbitos por causas externas e violência contra idosos: uma realidade velada. Rev Bras Promoç Saúde [Internet]. 2019 [acesso em 2020 Mar 2];32:8685. Disponível em: https://periodicos.unifor.br/RBPS/article/view/8685/pdf

25. Pereira SG, Santos CB, Doring M, Portella MR. Prevalence of household falls in long-lived adults and association with extrinsic factors. Rev Latino Am Enferm [Internet]. 2017 [acesso em 2020 Mar 2];25:e2900. Disponível em: https://www.scielo.br/scielo.php?pid=S0104-11692017000100382\&script=sci_abstract

26. Charles A, Buckinx F, Locquet M, Reginster JY, Petermans J, Gruslin B, et al. Prediction of Adverse Outcomes in Nursing Home Residents According to Intrinsic Capacity Proposed by the World Health Organization. J Gerontology [Internet]. 2019 [acesso em 2020 Mar 2];75:1594-99. Disponível em: https://academic.oup.com/ biomedgerontology/article-abstract/75/8/1594/5575908?redirectedFrom=fulltext

27. Oliveira T, Baixinho CL, Henriques MA. Risco multidimensional de queda em idosos. Rev Bras Promoç Saúde [Internet]. 2018 [acesso em 2020 Mar 2];31(2):1-9. Disponível em: https://periodicos.unifor.br/RBPS/article/ view/7058

28. Neves ÁQ, Silva AMC, Cabral JF, Mattos IE, Santiago LM. Prevalence of and factors associated with frailty in elderly users of the Family Health Strategy. Rev Bras Geriatr Gerontol [Internet]. 2018 [acesso em 2020 Mar 2];21(6):680-90. Disponível em: https://www.scielo.br/scielo.php?pid=S180998232018000600680\&script=sci_abstract 
29. Gullich I, Cordova DDP. Falls in the elderly: a population-based study. Rev Soc Bras Clin Med [Internet]. 2017 [acesso em 2020 Mar 2];15(4):230-4. Disponível em: http://docs.bvsalud.org/ biblioref/2018/01/877065/154230-234.pdf

30. Mamani ARN, Reines AAO, Azevedo RCS, Vechia ADRD, Segri NJ, Cardoso JDC. Cuidador de idosos: conhecimentos, atitudes e práticas sobre quedas e sua prevenção. Rev Bras Enferm [Internet]. 2019 [acesso em 2020 Mar 2];72(supl. 2):119-126. Disponível em: https://www.scielo.br/scielo.php?pid=S003471672019000800119\&script=sci_arttext\&tIng=pt

\section{Endereço para correspondência:}

Samya Regina Nunes Oliveira

Universidade Estadual do Ceará

Av. Silas Munguba, 1700

Bairro: Campus do Itaperi

CEP: 60714-903 - Fortaleza - CE - Brasil

E-mail: samya.regina@aluno.uece.br

Como citar: Oliveira SRN, Messias FML, Cândido JAB, Torres GMC, Figueiredo IDT, Pinto AGA, et al. Fatores associados a quedas em idosos: inquérito domiciliar. Rev Bras Promoç Saúde. 2021;34:10998. 